\title{
COSMIC RAY ANTIMATTER FROM SUPERSYMMETRIC DARK MATTER
}

\author{
John S. HAGELIN \\ Department of Physics, Maharishi International University, Fairfield, IA 52550, USA \\ Gordon L. KANE \\ Randall Laboratory of Physics, University of Michigan, Ann Arbor, MI 48109, USA
}

Received 17 April 1985

(Revised 29 July 1985)

\begin{abstract}
In this paper we study the antiproton, positron and gamma-ray fluxes resulting from the annihilation of supersymmetric dark matter in our galactic halo. We show that the requirement of closure imposed by inflationary cosmologies constrains the supersymmetric dark matter to be either a relatively pure photino eigenstate or a relatively pure higgsino eigenstate, or possibly a scalar neutrino with a mass less than $4 \mathrm{GeV}$. The photino choice can lead to observable $\bar{p}$ fluxes when sfermion masses are $\leq 50-60 \mathrm{GeV}$. Such a scenario is testable via the radiative production of photinos at PEP and PETRA. The higgsino scenario leads to observable $\bar{p}$ fluxes independent of sfermion masses, provided $\left\langle 0\left|H_{0}^{1}\right| 0\right\rangle /\left\langle 0\left|H_{0}^{2}\right| 0\right\rangle=v_{1} / v_{2} \geq 2$. In contrast, sneutrino dark matter annihilates almost entirely into neutrinos, resulting in a monochromatic neutrino flux that is probably unobservable.
\end{abstract}

In a recent paper [1], Silk and Srednicki (S\&S) show that cosmic ray antiprotons, positrons and gamma-rays can provide an experimental approach to the study of supersymmetric dark matter concentrated in our galactic halo. They consider the interesting and important case where the supersymmetric dark matter candidate is a photino, and show for a particular choice of sfermion masses that the annihilation of relic photinos in the halo can lead to observable $\overline{\mathrm{p}}, \mathrm{e}^{+}$and $\gamma$-ray fluxes. In this paper, we extend the analysis of $\mathrm{S} \& \mathrm{~S}$ to the most general supersymmetric dark matter candidate. We show that the requirement of closure imposed by inflationary cosmologies places a powerful constraint on the structure of the minimal supersymmetric model, which forces the dark matter candidate to be either a relatively pure photino eigenstate or a relatively pure higgsino eigenstate, or possibly a scalar neutrino. We consider each of these cases and compare their cosmic ray signatures.

In an inflationary cosmology, the mean mass density of the universe $\rho$ must be very nearly equal to the closure density $\rho_{\mathrm{cr}}=3 H_{0}^{2} / 8 \pi G$, where $H_{0}$ is the Hubble 
constant [2]. Since baryonic matter appears to contribute at most $0.2 \rho_{\mathrm{cr}}$, most of the mass density of the universe must be in the form of nonbaryonic matter [3].

Supersymmetric theories offer a natural framework for dark matter candidates because, in many models, the lightest supersymmetric particle (LSP) is absolutely stable. By "supersymmetric particle," we mean the superpartners of any ordinary particles: gauge fermions (photino, gluinos, winos, etc.), Higgs fermions (higgsinos), scalar quarks and leptons (squarks and sleptons), and the gravitino. In the very early universe, all these particles would be present in thermal equilibrium. As the temperature falls, the heavier supersymmetric particles decay into the lighter ones. Eventually, only the LSP will be left. It can disappear only by pair annihilation. In an inflationary cosmology, one must require that the rate of annihilation be such that the present day mass density is equal to the closure density, which we take to be between $(0.5-2.0) \times 10^{-29} \mathrm{~g} / \mathrm{cm}^{3}$, corresponding to a Hubble constant $H_{0}$ between (50-100) $\mathrm{kms}^{-1} \mathrm{Mpc}^{-1}$, although one could take the point of view that $H_{0} \simeq 100$ $\mathrm{kms}^{-1} \mathrm{Mpc}^{-1}$ is disfavored by constraints on the ages of globular clusters [4].

Previous cosmological and phenomenological analyses $[5,6]$ have shown that the most likely candidate for the LSP is a neutral gaugino-higgsino particle ("neutralino" or " $\chi$ ") or possibly a scalar neutrino ("sneutrino" or " $\tilde{\nu}$ "). We shall explore these two possibilities in turn.

In our analysis, we shall consider a minimal supersymmetric model with two light doublets of Higgs chiral superfields $H_{1}$ and $H_{2}$ of weak hypercharge \pm 1 respectively [7]. The mass matrices for the charged and neutral supersymmetric fermions - gauginos and higgsinos - are determined by the lagrangian terms

$$
\varrho \exists \varepsilon \varepsilon_{i j} \tilde{H}_{1}^{i} \tilde{H}_{2}^{j}-M_{2} \tilde{W}_{a} \tilde{W}_{a}-M_{1} \tilde{B} \tilde{B}
$$

where $W_{a}$ and $B$ denote $\mathrm{SU}(2)$ and $\mathrm{U}(1)$ gauge superfields respectively, the tildes denote fermionic components and $i, j,(a)$ are doublet (triplet) SU(2) indices. We generally expect the parameters $\varepsilon, M_{1}, M_{2}$ to be $\mathrm{O}\left(M_{\mathrm{W}}\right)$, though it is conceivable that $\varepsilon$ might be very much smaller [8]. We shall assume

$$
M_{1}=\frac{5}{3} \frac{\alpha_{1}}{\alpha_{2}} M_{2} \text {, }
$$

where $\alpha_{i}=g_{i} / 4 \pi, i=1,2,3$ are the gauge coupling constants, which holds to leading order in the renormalization group equations if $\mathrm{SU}(2) \times \mathrm{U}(1)$ is eventually embedded in a unifying non-abelian group [7]. In the neutral sector there are at least four supersymmetric fermions which mix, namely the $\tilde{W}^{3}, \tilde{B}^{0}, \tilde{H}_{1}^{0}$ and $\tilde{H}_{2}^{0}$. When the conventional Higgs-gauge field couplings are combined with the lagrangian terms 
(1), their mixing matrix is

$$
\left(\tilde{W}^{3}, \tilde{B}^{0}, \tilde{H}_{1}^{0}, \tilde{H}_{2}^{0}\right)\left(\begin{array}{cccc}
M_{2} & 0 & -\sqrt{\frac{1}{2}} g_{2} v_{1} & \sqrt{\frac{1}{2}} g_{2} v_{2} \\
0 & \frac{5}{3} \frac{\alpha_{1}}{\alpha_{2}} M_{2} & \sqrt{\frac{1}{2}} g_{1} v_{1} & -\sqrt{\frac{1}{2}} g_{1} v_{2} \\
-\sqrt{\frac{1}{2}} g_{2} v_{1} & \sqrt{\frac{1}{2}} g_{1} v_{1} & 0 & \varepsilon \\
\sqrt{\frac{1}{2}} g_{2} v_{2} & \sqrt{\frac{1}{2}} g_{1} v_{2} & \varepsilon & 0
\end{array}\right)\left(\begin{array}{c}
\tilde{W}^{3} \\
\tilde{B}^{0} \\
\tilde{H}_{1}^{0} \\
\tilde{H}_{2}^{0}
\end{array}\right),
$$

where $\left\langle 0\left|H_{1,2}^{0}\right| 0\right\rangle \equiv v_{1,2}$ with $M_{\mathrm{w}}^{2}=\frac{1}{2} g_{2}^{2}\left(v_{1}^{2}+v_{2}^{2}\right)$.

The general form of the orthogonal rotation that diagonalizes the matrix (3) is quite complicated and the Majorana mass eigenstate fields $\chi_{i}$ are in general complicated combinations of $\tilde{W}^{3}, \tilde{B}^{0}, \tilde{H}_{1}^{0}$ and $\tilde{H}_{2}^{0}$ :

$$
\chi_{i} \equiv\left(\alpha_{i} \tilde{W}^{3}+\beta_{i} \tilde{B}^{0}+\gamma_{i} \tilde{H}_{1}^{0}+\delta_{i} \tilde{H}_{2}^{0}\right) .
$$

In the limit $\varepsilon \rightarrow 0$ there is a light higgsino eigenstate

$$
\tilde{S}^{0} \equiv \frac{v_{2} \tilde{H}_{1}^{0}+v_{1} \tilde{H}_{2}^{0}}{v}, \quad M_{\tilde{\mathrm{S}}^{0}}=2 v_{1} v_{2} \varepsilon / v^{2},
$$

where we have introduced $v=\sqrt{v_{1}^{2}+v_{2}^{2}}$. In the limit of $M_{2} \rightarrow 0$ there is a light photino eigenstate:

$$
\tilde{\gamma} \equiv \frac{g_{1} \tilde{W}^{3}+g_{2} \tilde{B}^{0}}{\sqrt{g_{1}^{2}+g_{2}^{2}}}, \quad M_{\tilde{\gamma}}=\frac{8}{3} \frac{g_{1}^{2}}{g_{1}^{2}+g_{2}^{2}} M_{2} .
$$

If $M_{2}$ and $\varepsilon$ are both small, the remaining two mass eigenstates are

$$
\tilde{Z}_{ \pm}^{0} \equiv \frac{g_{1} \tilde{B}^{0}-g_{2} \tilde{W}^{3} \pm \sqrt{g_{1}^{2}+g_{2}^{2}} \tilde{A}^{0}}{\sqrt{2\left(g_{1}^{2}+g_{2}^{2}\right)}}, \quad M_{\tilde{Z}_{ \pm}^{0}}=M_{\mathrm{Z}^{0}}=\sqrt{\frac{1}{2}\left(g_{1}^{2}+g_{2}^{2}\right)} v
$$

where $\tilde{A}^{0}$ is the higgsino combination orthogonal to $\tilde{S}^{0}$ (eq. (5)).

In the case where the LSP is a neutralino, the requirement of closure imposed by inflation places a powerful constraint on the parameters $M_{2}, \varepsilon$ in the lagrangian (1), on which the neutralino mass and annihilation cross section depend. To proceed with a quantitative analysis of this cosmological constraint, we recall the results of the cosmological analysis of ref. [5], which is applicable to the most general 
neutralino $\chi$ :

$$
\rho_{\chi}=4.0 \times 10^{-40}\left(\frac{T_{\chi}}{T_{\gamma}}\right)^{3}\left(\frac{T_{\gamma}}{2.8^{\circ} \mathrm{K}}\right)^{3} N_{\mathrm{F}}^{1 / 2}\left(\frac{\mathrm{GeV}^{-2}}{\tilde{a} x_{\bar{y}}+\frac{1}{2} \tilde{b} x_{\tilde{\sigma}}^{2}}\right) \frac{\mathrm{g}}{\mathrm{cm}^{3}},
$$

where $x_{\bar{G}} \equiv k T_{\bar{F}} / m_{\chi}$ with $T_{\bar{G}}$ the freeze-out temperature for $\chi \chi$ annihilation [generally $\left.x_{\bar{\varphi}}=0\left(\frac{1}{20}\right)\right]$, and $\left(T_{\chi} / T_{\gamma}\right)^{3}$ accounts for the subsequent reheating of the photon temperature relative to the temperature of $\chi$ due to the annihilation of particles with $m<x_{\mathfrak{G}} m_{\chi}$. Typical values of $\left(T_{\chi} / T_{\gamma}\right)^{3}$ and of the number $N_{\mathrm{F}}$ of effective degrees of freedom are tabulated on p. 467 of ref. [5]. The parameters $\tilde{a}$ and $\tilde{b}$ are determined by the particular model according to:

$$
\begin{aligned}
& \tilde{a} \equiv \sum_{\mathrm{f}} \theta\left(m_{\chi}-m_{\mathrm{f}}\right) \frac{1}{2 \pi} \frac{p}{m_{\chi}} m_{\mathrm{f}}^{2}\left(A_{\mathrm{f}}-B_{\mathrm{f}}\right)^{2}, \\
& \tilde{b} \equiv \sum_{\mathrm{f}} \theta\left(m_{\chi}-m_{\mathrm{f}}\right) \frac{1}{2 \pi} \frac{p}{m_{\chi}}\left[\left(A_{\mathrm{f}}^{2}+B_{\mathrm{f}}^{2}\right)\left(4 m_{\chi}^{2}-m_{\mathrm{f}}^{2}\right)+6 A_{\mathrm{f}} B_{\mathrm{f}} m_{\mathrm{f}}^{2}\right],
\end{aligned}
$$

where $p \equiv \sqrt{m_{\chi}^{2}-m_{\mathrm{f}}^{2}}$ and

$$
\begin{aligned}
& A_{\mathrm{f}}=\left(\gamma^{2}-\delta^{2}\right) \frac{g_{1} \sin \theta_{\mathrm{W}}+g_{2} \cos \theta_{\mathrm{W}}}{4 M_{\mathrm{Z}^{0}}^{2}}\left(\frac{1}{2} Y_{\mathrm{f}_{\mathrm{L}}} g_{1} \sin \theta_{\mathrm{w}}-T_{\mathrm{f}_{\mathrm{L}}}^{3} g_{2} \cos \theta_{\mathrm{w}}\right) \\
& +\frac{\left(T_{\mathrm{f}_{\mathrm{L}}}^{3} \alpha g_{2}+\frac{1}{2} Y_{\mathrm{f}_{\mathrm{L}}} \beta g_{1}\right)^{2}}{2 m_{\tilde{\mathrm{f}}_{\mathrm{L}}}^{2}}- \begin{cases}\gamma^{2} m_{\mathrm{f}}^{2} / 4 v_{1}^{2} m_{\tilde{\mathrm{f}}_{\mathrm{R}}}^{2}, & \mathrm{u}, \mathrm{c}, \mathrm{t} \\
\delta^{2} m_{\mathrm{f}}^{2} / 4 v_{2}^{2} m_{\tilde{\mathrm{f}}_{\mathrm{R}}}^{2}, & \mathrm{e}, \mu, \tau, \mathrm{d}, \mathrm{s}, \mathrm{b},\end{cases} \\
& B_{\mathrm{f}}=\left(\gamma^{2}-\delta^{2}\right) \frac{g_{1} \sin \theta_{\mathrm{W}}+g_{2} \cos \theta_{\mathrm{W}}}{8 M_{\mathrm{Z}^{0}}^{2}} Y_{\mathrm{f}_{\mathrm{R}}} g_{1} \sin \theta_{\mathrm{W}} \\
& -\frac{\left(\frac{1}{2} Y_{\mathrm{f}_{R}} \beta g_{1}\right)^{2}}{2 m_{\tilde{\mathrm{f}}_{\mathrm{R}}}^{2}}+ \begin{cases}\gamma^{2} m_{\mathrm{f}}^{2} / 4 v_{1}^{2} m_{\tilde{\mathrm{f}}_{\mathrm{L}}}^{2}, & \mathrm{u}, \mathrm{c}, \mathrm{t} \\
\delta^{2} m_{\mathrm{f}}^{2} / 4 v_{2}^{2} m_{\tilde{\mathrm{f}}_{\mathrm{L}}}^{2}, & \text { e, } \mu, \tau, \mathrm{d}, \mathrm{s}, \mathrm{b},\end{cases}
\end{aligned}
$$

where $\tilde{f}_{L, R}$ are the spartners of the left-(right-)handed fermions $f$ with electromagnetic charge $Q_{\mathrm{f}}$.

We see that the present mass density $(8)$ depends through $(9,10)$ on the parameters $M_{2}, \varepsilon$ in the lagrangian (1) which, together with the ratio $v_{1} / v_{2}$, determine the mass and content of the neutralino mass eigenstates. A numerical search of the space of parameters $M_{2}, \varepsilon, v_{1} / v_{2}$ reveals that the closure constraint $\rho_{\chi}=(0.5-2.0) \times 10^{-29}$ $\mathrm{g} / \mathrm{cm}^{3}$ forces $M_{2}, \varepsilon$ into a domain where the LSP neutralino is either a relatively 
pure photino eigenstate or a relatively pure higgsino eigenstate. This conclusion can also be inferred directly from the diagrams of ref. [5].

The fundamental importance of this conclusion is that it greatly simplifies the phenomology of the LSP for both particle physics and astrophysics. Without the constraint of closure imposed by inflationary cosmologies, the lightest neutralino could be an almost arbitrary admixture of gaugino and higgsino [5]. In contrast, as a result of inflation, the possibilities get restricted to either a photino or a higgsino eigenstate, with the phenomenological properties of these particles determined almost entirely by their masses, which are themselves constrained by the requirement of closure. This leads to a rather definite set of predictions for cosmic ray fluxes due to the annihilation of relic neutralinos, as well as for terrestrial particle physics experiments. We shall now discuss the cosmic ray phenomenology of the photino and higgsino dark matter candidates in turn.

One simplifying feature of the photino choice is that it is independent of the ratio of vacuum expectation values $v_{1} / v_{2}$, since this ratio does not significantly affect the composition or the phenomenology of the light gaugino eigenstate of the matrix (3). It is, on the other hand, necessary to choose values for the squark and slepton masses, since these masses affect the gaugino annihilation cross section, which proceeds predominantly via sfermion exchange.

Many supersymmetric models [7,9] place squark and slepton masses in the 50-100 GeV range, with squark masses possibly heavier than slepton masses. If we choose as an initial example $M_{\tilde{\mathrm{q}}}=100 \mathrm{GeV}$ and $M_{\hat{\ell}}=50 \mathrm{GeV}$ and take $\rho_{\mathrm{cr}}=5 \times$ $10^{-30} \mathrm{~g} / \mathrm{cm}^{3}$ (corresponding to a Hubble constant $H_{0}=50 \mathrm{kms}^{-1} \mathrm{Mpc}^{-1}$ ), the closure constraint requires a photino mass of $M_{\tilde{\gamma}}=4.2 \mathrm{GeV}$ and a present day (cold) annihilation cross section of $\left\langle\sigma v_{\text {rel }}\right\rangle_{\text {cold }}=8.5 \times 10^{-29} \mathrm{~cm}^{3} / \mathrm{s}$, with $97 \%$ of photinos annihilating into $\bar{\tau} \tau$ pairs and approximately $3 \%$ annihilating into $\bar{c} c$ (see table 1 , \#4).

The relative branching fractions for photino annihilation via $\bar{\tau} \tau, \bar{c} c$, etc. into final state antiprotons, positrons and gamma-rays can be estimated from experimental results [10] on $\mathrm{e}^{+} \mathrm{e}^{-} \rightarrow \bar{\tau} \tau, \overline{\mathrm{c}} \mathrm{c}$, etc. as in $\mathrm{S} \& \mathrm{~S}$ :

$$
\begin{aligned}
& \chi \chi \rightarrow \bar{\tau} \tau \rightarrow 1.5\left(\mathrm{e}^{+} \mathrm{e}^{-}\right)+0(\overline{\mathrm{p}} \mathrm{p})+1 \gamma+5.5 \nu, \\
& \chi \chi \rightarrow \overline{\mathrm{c}} \mathrm{c} \rightarrow 4\left(\mathrm{e}^{+} \mathrm{e}^{-}\right)+0.2(\overline{\mathrm{p}} \mathrm{p})+7 \gamma+22 \nu, \\
& \chi \chi \rightarrow \overline{\mathrm{b}} \mathrm{b} \rightarrow 7.5\left(\mathrm{e}^{+} \mathrm{e}^{-}\right)+0.3(\overline{\mathrm{p}} \mathrm{p})+13 \gamma+41 \nu .
\end{aligned}
$$

We will also adopt the following reasonable estimates of S\&S for the energy distributions of the final state particles:

(i) The energy of a final state $\gamma$ is comparable to the kinetic energy of a $\mathrm{p}$ or $\overline{\mathrm{p}}$ coming from the same channel, while the energy of a final state $\mathrm{e}^{ \pm}$or $\nu$ is typically half as large. 


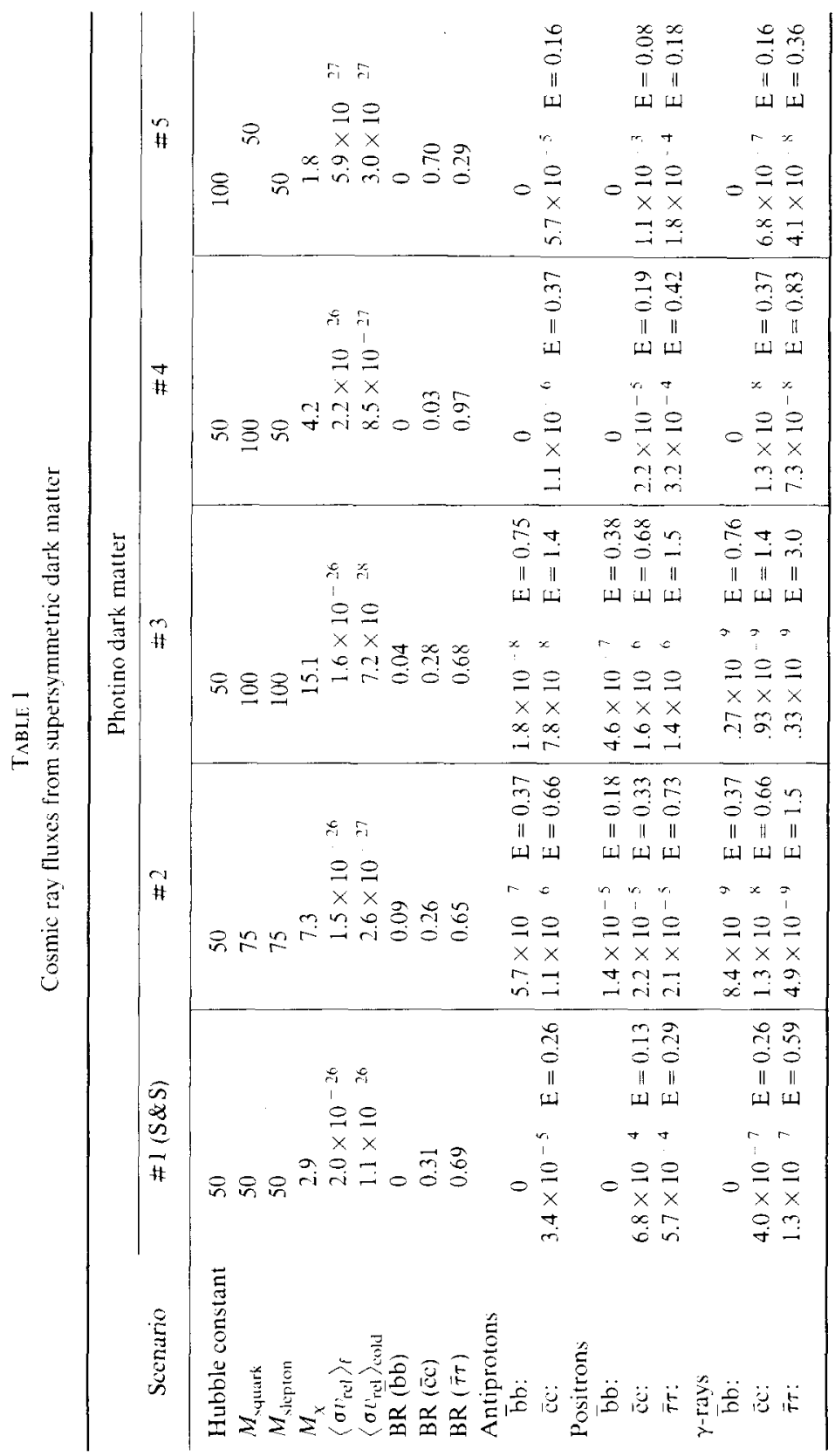




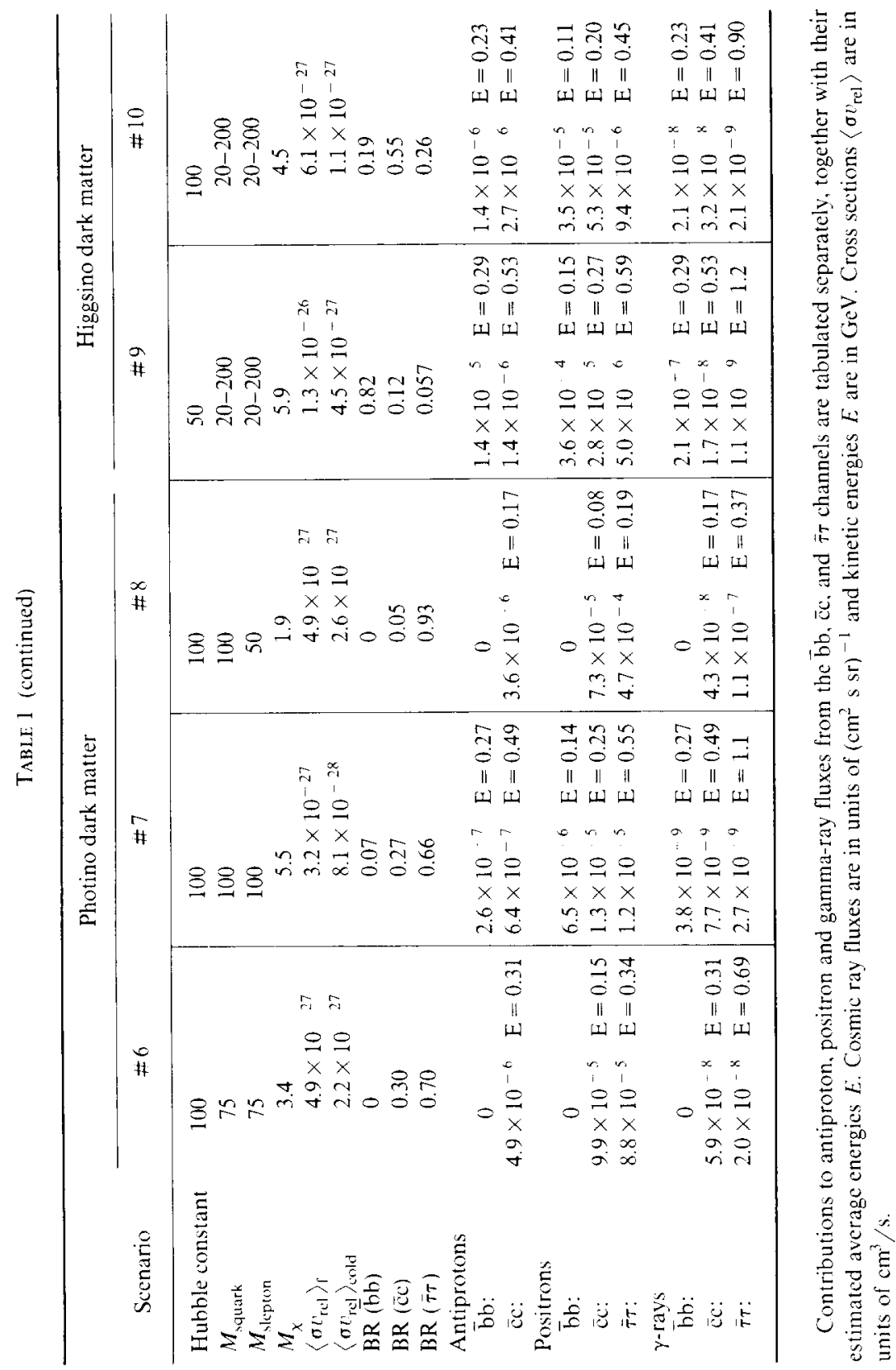


(ii) Decay products of the $\tau$ have the highest average kinetic energies with $E_{\gamma}^{\tau} \approx 0.2 M_{\chi}$, while $E_{\gamma}^{\mathrm{c}} \approx 0.09 M_{\chi}$ and $E_{\gamma}^{\mathrm{b}} \approx 0.05 M_{\chi}$.

The magnitudes of the cosmic ray fluxes depend critically on assumptions regarding the accretion of supersymmetric dark matter into our galactic halo and the trapping of cosmic rays in the halo by magnetic field irregularities. S\&S have shown that without such enhancements cosmic ray fluxes from relic photinos are too small to be interesting.

However, neutralinos and scalar neutrinos would have been "cold" at a time when the cosmological horizon contained a mass comparable to that of a galaxy, so one can expect these particles would have clustered in galaxies [5,6]. It therefore seems natural to assume that the supersymmetric LSP, in addition to providing closure density, comprises the massive dark matter in the galactic halo. Following this assumption, $S \& S$ choose a specific dynamical model [11] to derive an expression for the $\gamma$-ray flux in terms of a rotation velocity $V$ and core radius $a$ :

$$
\begin{aligned}
F_{\gamma}= & 1.7 \times 10^{-7}\left[\left\langle\boldsymbol{\sigma} v_{\mathrm{rel}}\right\rangle_{\gamma} /\left(10^{-26} \mathrm{~cm}^{3} \mathrm{~s}^{-1}\right)\right]\left[V /\left(200 \mathrm{~km} \mathrm{~s}^{-1}\right)\right]^{4} \\
& \times(a / 10 \mathrm{kpc})^{-3}\left(M_{\tilde{\gamma}} / 3 \mathrm{GeV}\right)^{-2}\left(\mathrm{~cm}^{2} \mathrm{~s} \mathrm{sr}\right)^{-1}
\end{aligned}
$$

Accepting these canonical values for the rotation velocity and core radius, our previously specified photino scenario yields a flux of $F_{\gamma}=0.9 \times 10^{-7}\left(\mathrm{~cm}^{2} \mathrm{~s} \mathrm{sr}\right)^{-1}$. Given the strong dependence of (12) on $V$ and on $a$, this flux should presumably be considered to be an order-of-magnitude estimate.

The $\gamma$-ray energy distribution is more accurately determined. In our sample scenario, we have a $4.2 \mathrm{GeV}$ photino annihilating $97 \%$ of the time into $\bar{\tau} \tau$ and $\approx 3 \%$ into $\overline{\mathrm{c}}$. Thus, despite the relatively larger branching ratio for photons from charm decay (11), the $\gamma$-ray flux from annihilating photinos is $85 \%$ from the $\tau$ channel. We therefore expect that the $\gamma$-ray spectrum should be broadly peaked at an energy $E \approx 0.2 M_{\tilde{\gamma}}$, or about $0.8 \mathrm{GeV}$ in this case. As pointed out by $\mathrm{S} \& \mathrm{~S}$, this spectrum is sufficiently hard that it could stand out against the observed isotropic $\gamma$-ray spectrum, which drops sharply above $100 \mathrm{MeV}$.

Still more interesting are cosmic ray antiprotons, which are trapped in the halo by magnetic field irregularities for a time $t_{\mathrm{h}}$, leading to an enhancement of the antiproton flux by a factor $\sim c t_{\mathrm{h}} / a$. This results in an expected flux of [1]:

$$
\begin{aligned}
F_{\overline{\mathrm{p}}}= & 5 \times 10^{-4}\left[\left\langle\sigma v_{\mathrm{rel}}\right\rangle_{\overline{\mathrm{p}}} /\left(10^{-26} \mathrm{~cm}^{3} \mathrm{~s}^{-1}\right)\right]\left[V /\left(200 \mathrm{~km} \mathrm{~s}^{-1}\right)\right]^{4} \\
& \times(a / 10 \mathrm{kpc})^{-2}\left(M_{\tilde{\gamma}} / 3 \mathrm{GeV}\right)^{-2}\left(K / 10^{+29} \mathrm{~cm}^{2} \mathrm{~s}^{-1}\right)^{-1} \\
& \times\left(\mathrm{cm}^{2} \mathrm{~s} \mathrm{sr}\right)^{-1},
\end{aligned}
$$

where $K$ is the diffusion constant for cosmic rays in the halo [12]. In our sample 
scenario, the antiproton flux comes entirely from charm decays, since $\tau$ decays do not yield antiprotons. Using (13), the anticipated flux is $1.1 \times 10^{-6}\left(\mathrm{~cm}^{2} \mathrm{~s} \mathrm{sr}\right)^{-1}$, however, one must bear in mind that uncertainties connected with cosmic ray trapping have been added to the previous uncertainties regarding photino clustering. The energy distribution of these cosmic ray antiprotons is expected to peak at around $0.4 \mathrm{GeV}$.

There are already some indications that cosmic ray antiprotons have been observed below the kinematic threshold for secondary production of about $2 \mathrm{GeV}$, with $F_{\overline{\mathrm{p}}}$ reported to be around $3 \times 10^{-6}\left(\mathrm{~cm}^{2} \mathrm{~s} \mathrm{sr}\right)^{-1}$ between 0.6 and $1.2 \mathrm{GeV}$ [13]. Our sample estimate lies somewhat below this value and considerably below the estimate of S\&S principally because our choice of squark and slepton masses favor $\tilde{\gamma} \tilde{\gamma} \rightarrow \bar{\tau} \tau$, which does not result in antiprotons. We will present a more complete range of scenarios following this discussion of general principles.

The positron flux is also given by (13), but the multiplicity of positrons in the final state (11) is much larger than for antiprotons. In particular, our sample scenario predicts a positron flux of $F_{\mathrm{c}^{+}} \approx 3 \times 10^{-4}\left(\mathrm{~cm}^{2} \mathrm{~s} \mathrm{sr}\right)^{-1}$ primarily through the $\tau$ channel, with an energy distribution centered around $0.4 \mathrm{GeV}$. This is in good qualitative agreement with the $\mathrm{e}^{+}$flux inferred from the measured [14] ratio $\mathrm{e}^{+} /\left(\mathrm{e}^{+}+\mathrm{e}^{-}\right)$and the synchrotron emissivity of the galaxy $[1,15]$ :

$$
F_{\mathrm{e}^{+}} \approx 1 \times 10^{-3}\left(\mathrm{~cm}^{2} \mathrm{~s} \mathrm{sr}\right)^{-1} \quad(0.2 \leq E \leq 1 \mathrm{GeV})
$$

We conclude our cosmic ray analysis of the photino LSP scenario with a table in which the $\overline{\mathrm{p}}, \mathrm{e}^{+}$and $\gamma$-ray fluxes are summarized for selected values of the sfermion masses and the Hubble constant (table 1). The table lists the sfermion masses, the photino mass required to reproduce the closure density, the annihilation cross section at freeze-out $\left\langle\sigma v_{\text {rel }}\right\rangle_{\text {, }}$, the annihilation cross section for cold photinos $\left\langle\sigma v_{\text {rel }}\right\rangle_{\text {cold }}$ together with the branching fractions for annihilation into $\bar{\tau} \tau, \bar{c} c$ and $\bar{b} b$, and the separate contributions to the $\gamma$-ray, $\overline{\mathrm{p}}$ and $\mathrm{e}^{+}$fluxes from the $\bar{\tau} \tau, \overline{\mathrm{c}} \mathrm{c}$ and $\overline{\mathrm{b}} \mathrm{b}$ channels along with their expected average energies.

We note that the $\bar{b} b$ channel contribution to photino annihilation is always small due to an electric charge suppression, despite the $M_{\mathrm{f}}^{2}$ dependence of the cold annihilation cross section on the final state fermion mass. The relative importance of $\bar{c}$ versus $\bar{\tau} \tau$ depends critically on the relative squark and slepton masses. We also note that in general, heavier sfermion masses suppress the annihilation cross section which forces the photino to be more massive. This results in a strong suppression of the cosmic ray fluxes, which depend not only on the annihilation cross section but on the squared photino number density, which is inversely proportional to the photino mass for a fixed halo density. On the other hand, choosing a larger Hubble constant (e.g. $100 \mathrm{kms}^{-1} \mathrm{Mpc}^{-1}$ ) and a correspondingly larger critical density $\left.\left(2 \times 10^{-29}\right) \mathrm{g} / \mathrm{cm}^{3}\right)$ permits the photino to be lighter. This results in a larger halo number density and hence larger cosmic ray fluxes, though the energy distributions 
are considerably softer. It follows that the most optimistic photino scenario for cosmic ray antiprotons is scenario \# 1 (selected by $S \& S$ ), where light squark masses result in a large $\bar{c} c$ channel contribution to photino annihilation, leading to a large $\overline{\mathrm{p}}$ yield. Here light sfermion masses also result in a large annihilation cross section and hence a light photino, both of which contribute to a large predicted flux. Scenario \# 5 with $H_{0}=100$ and scenarios \#2 and \#6 with sfermion masses of $75 \mathrm{GeV}$ and $H_{0}=50$ and 100 respectively also show interesting $\overline{\mathrm{p}}$ fluxes (see table 1 ). Positron fluxes and energy distributions appear to be consistent with experimental data (14) in scenarios \#1, \#4 and possibly \#5 and \#8-scenarios that are also the most likely to result in observable $\gamma$-ray fluxes.

We now turn to the consideration of the higgsino LSP scenario. Here the situation is simplified by the fact that higgsino annihilation is dominated by direct-channel $Z^{0}$ exchange, so that sfermion masses are all but irrelevant. There is now, however, a dependence on the ratio of vacuum expectation values $v_{1} / v_{2}$ which impacts the composition of the higgsino eigenstate (5), but we find that the physics is very insensitive to $v_{1} / v_{2}$ as long as $v_{1} / v_{2} \geq 2$. [One might expect $v_{1}>v_{2}$ since $v_{1}$ gives mass to the top quark and $v_{2}$ gives mass to the bottom quark]. However, if $v_{1} / v_{2} \rightarrow 1$, the dominant $\left(\mathrm{Z}^{0}\right)$ contribution to higgsino annihilation vanishes, and consistency with cosmology forces the higgsino to be very heavy. This we have argued leads to uninterestingly small predictions for cosmic ray fluxes, so we will confine our discussion to the case where $v_{1} / v_{2}>2$, where this ratio ceases to be a pertinant parameter.

With this one restrictive assumption, the higgsino LSP scenario is both simple and phenomenologically promising. For a Hubble constant of $50 \mathrm{kms}^{-1} \mathrm{Mpc}^{1}$ we obtain a higgsino mass of $5.9 \mathrm{GeV}$, which is very insensitive to sfermion masses. This is sufficiently far above the b-quark threshold that the b channel accounts for $82 \%$ of higgsino annihilations (due to the $M_{\mathrm{f}}^{2}$ dependence of the cold annihilation cross section). $\gamma$-ray, $\overline{\mathrm{p}}$ and $\mathrm{e}^{+}$fluxes are all dominated by their $\overline{\mathrm{b}} \mathrm{b}$ channel contributions (see table 1, \#9). Since the annihilation is essentially into b quarks at rest, the final state $\overline{\mathrm{p}}, \mathrm{e}^{+}$and $\gamma$-ray spectra will be essentially the same as for $\mathrm{e}^{+} \mathrm{e}^{-} \rightarrow \overline{\mathrm{b}} \mathrm{b} \rightarrow \overline{\mathrm{p}} \mathrm{X}$, etc. at CESR [16]. This $\overline{\mathrm{p}}$ spectrum falls off with energies $E_{\overline{\mathrm{p}}}>300 \mathrm{MeV}$ and presumably peaks at $E_{\overline{\mathrm{p}}} \approx 300 \mathrm{MeV}$. Numerically, we estimate using (12) and (13) that $F_{\overline{\mathrm{p}}} \approx 1.5$ $\times 10^{-5}\left(\mathrm{~cm}^{2} \mathrm{~s} \mathrm{sr}\right)^{-1}, F_{\mathrm{e}^{+}} \approx 4 \times 10^{-4}\left(\mathrm{~cm}^{2} \mathrm{~s} \mathrm{sr}\right)^{-1}$, and $F_{\gamma} \approx 2 \times 10^{-7}\left(\mathrm{~cm}^{2} \mathrm{~s} \mathrm{sr}\right){ }^{-1}$, where the uncertainties associated with these numbers due to the accretion of higgsinos and trapping of cosmic rays in the halo have been discussed previously. We see that for cosmic ray phenomenology the higgsino scenario compares favorably with the most optimistic photino scenario.

If we choose a larger value for the Hubble constant, $H_{0}=100 \mathrm{kms}^{-1} \mathrm{Mpc}^{-1}$, the higgsino mass drops almost to the b-quark threshold (see table $1, \# 10$ ). Thus, as a result of phase space, the $\bar{b} b$ channel accounts for only $19 \%$ of higgsino annihilations, while the $\overline{\mathrm{c}} \mathrm{c}$ channel accounts for about $55 \%$. The cold annihilation cross section is reduced to about one quarter of its value in the $H_{0}=50\left(\mathrm{kms}^{1} \mathrm{Mpc}^{-1}\right)$ 
scenario, and the $\gamma$-ray, $\overline{\mathrm{p}}$ and $\mathrm{e}^{+}$fluxes are all suppressed by a comparable fraction. The energy distribution is expected to be harder for the $\bar{c} c$ channel than for the $\bar{b} b$ channel, so this "large- $H_{0}$ " scenario is also of some phenomenological interest.

One point of possible importance is that although there are considerable uncertainties in the antiproton and positron fluxes associated with the accretion of dark matter and the trapping of cosmic rays in our galactic halo, the ratio of positrons to antiprotons is largely independent of these uncertainties. In the higgsino LSP scenario, this ratio is fairly stable due to the dominance of the $\bar{b} b$ and/or $\overline{c c}$ decay channels, and has a value of about 25 . In the photino scenario, this ratio varies between approximately 30 and 300 , depending on whether squark and slepton masses are taken to be degenerate or the squarks are chosen to be heavier.

We have shown that both the photino and the higgsino LSP scenarios can lead to observable $\overline{\mathrm{p}}, \mathrm{e}^{+}$, and $\gamma$-ray fluxes. Observable fluxes are somewhat more generic for the higgsino case, in which small sfermion masses are not required. The higgsino case, however, does require the ratio of vacuum expectation values $v_{1} / v_{2}$ to be $\geq 2$.

The primary means for distinguishing between the photino and higgsino LSP scenarios appears to be the $\mathrm{e}^{+} / \overline{\mathrm{p}}$ ratio since the absolute fluxes contain significant theoretical uncertainties. The $\mathrm{e}^{+} / \overline{\mathrm{p}}$ ratio is $\sim 25$ for the higgsino case and larger (possibly much larger) for the photino case. Presumably, in the future, as the absolute fluxes themselves become better determined theoretically, they will also provide a useful means for distinguishing between the two scenarios. We also note [17] that experiments over the next few years will achieve sensitivities one to two orders of magnitude better than ref. [13].

Also, we note that photino scenarios that are capable of generating observable cosmic ray fluxes should be verifiable in particle physics experiments. Since we have seen that a viable photino scenario requires a photino mass between $2-5 \mathrm{GeV}$ and relatively light $(\$ 50-60 \mathrm{GeV}$ ) sleptons, it should be possible to observe the radiative production of photinos in $\mathrm{e}^{+} \mathrm{e}^{-}$annihilation [18-20]. Since the phase-space suppression for a 2-5 GeV photino is small at PEP and PETRA energies, a dedicated experiment should be sensitive to selectron masses up to $60-70 \mathrm{GeV}$ $[18,19]$.

Finally we turn to a consideration of the sneutrino LSP scenario. Cosmologically, sneutrinos are very different from photinos and higgsinos because sneutrinos can pair-annihilate via neutralino exchange without P-wave or helicity suppression [6]. It follows that the sneutrino mass density today is approximately independent of $M_{\tilde{\nu}}$. This contrasts with the familiar neutralino case (or even the case of a heavy neutrino) where the annihilation cross section is proportional to $M_{\chi}^{2}$ or to a final state mass ${ }^{2}$. In the neutralino case, the cosmological mass density grows at least as $1 / M_{\chi}^{2}$ for small $M_{\chi}$, which leads to a rather precise determination of $M_{\chi}$, as we have shown.

The requirement of closure does nevertheless lead to a rather stringent upper bound on $M_{\tilde{v}}$ based on the following argument [6]. Unless the Majorana mass terms 
TABLE 2

Upper bound on $M_{\tilde{\nu}}$ from closure as a function of $v_{1} / v_{2}$ and the Hubble constant $H_{0}$.

\begin{tabular}{crr}
\hline$v_{1} / v_{2}$ & 50 & 100 \\
\hline 1 & $4 \mathrm{GeV}$ & $2 \mathrm{GeV}$ \\
2 & $2.5 \mathrm{GeV}$ & $1.3 \mathrm{GeV}$ \\
4 & $1 \mathrm{GeV}$ & $0.5 \mathrm{GeV}$ \\
\hline
\end{tabular}

$M_{2}, \varepsilon$ in the neutralino mass matrix (3) are very small, sneutrino annihilation via neutralino exchange is so efficient that $\rho_{\tilde{\nu}}$ is well below the closure density. But in the limit $M_{2}, \varepsilon \rightarrow 0$ there is a light photino (6) and light higgsino (5) eigenstate, neither of which contributes to sneutrino annihilation. In the same limit, there are two degenerate $\tilde{Z}_{ \pm}^{0}$ neutralinos (7) that give equal and opposite contributions to the annihilation amplitude. A non-zero value for $\varepsilon$ splits the degeneracy between the two $\tilde{Z}_{ \pm}^{0}$ eigenstates, causing the sneutrinos to annihilate very efficiently. The sneutrino mass density $\rho_{\hat{v}}$ is therefore a sensitive function of $\varepsilon$, and thus the requirement of closure implies a definite value for $\varepsilon$. However, $\varepsilon$ also determines the mass of the higgsino eigenstate $\tilde{S}^{0}$, and if the sneutrino is to be the LSP, it must have a

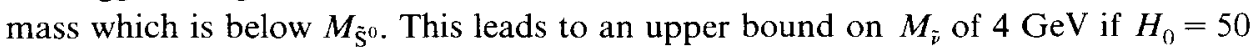
$\mathrm{kms}^{-1} \mathrm{Mpc}^{-1}$ and $v_{1} / v_{2}=1$, and an even stronger bound if $H_{0}$ and $/$ or $v_{1} / v_{2}$ is increased, as shown in table 2.

Such small values of $M_{\tilde{v}}$ are somewhat disfavored by today's most fashionable supersymmetric models, and are not suggested by existing bounds on the selectron mass from $\mathrm{e}^{+} \mathrm{e}^{-}$experiments [20]. Such stringent upper bounds on $M_{\tilde{\nu}}$ might therefore be considered as evidence against the sneutrino LSP scenario. However, in the absence of a compelling supersymmetric model, we will accept these upper bounds on $M_{\bar{\nu}}$ and entertain the sneutrino LSP as an interesting cosmological possibility.

The cosmic ray phenomenology of sneutrino dark matter is rather transcendental. The sneutrinos annihilate almost entirely into neutrinos via neutralino exchange. The annihilation cross section is completely determined by the choice of Hubble constant:

$$
\left\langle\sigma v_{\text {rel }}\right\rangle_{\text {cold }} \simeq \begin{cases}1.5 \times 10^{-26} \mathrm{~cm}^{3} \mathrm{~s}^{-1}, & H_{0}=50 \mathrm{kms}^{-1} \mathrm{Mpc}^{-1} \\ 3.7 \times 10^{-27} \mathrm{~cm}^{3} \mathrm{~s}^{-1}, & H_{0}=100 \mathrm{kms}^{-1} \mathrm{Mpc}^{-1}\end{cases}
$$

Since sneutrinos, like neutralinos, fall into the category of "cold" dark matter, they will aggregate in the galactic halo, and we can use an equation for the neutrino flux which is similar to that for $\gamma$-rays:

$$
F_{\nu}=1.7 \times 10^{-7}\left[2\left\langle\sigma v_{\text {rel }}\right\rangle_{\mathrm{cold}} / 10^{-26}\right]\left[M_{\bar{\nu}} / 3 \mathrm{GeV}\right]^{-2}\left(\frac{2}{4}\right)
$$


where the factor of two in square brackets counts two neutrinos produced per annihilation and the final factor of $\frac{2}{4}$ combines the flux from both $\tilde{\nu}$ and $\tilde{\tilde{\nu}}$ annihilation and then divides by four since the $\tilde{\nu}$ and the $\overline{\tilde{\nu}}$ each accounts for half of the total mass density and therefore half the number density, so the flux from each will be suppressed by $1 / n^{2}$ or $\frac{1}{4}$. This leads to a combined $\nu, \bar{\nu}$ flux in $\left(\mathrm{cm}^{2} \mathrm{~s} \mathrm{sr}\right)^{-1}$ of

$$
F_{\nu, \bar{\nu}} \simeq \begin{cases}2.3 \times 10^{-6} / M_{\tilde{\nu}}^{2}\left(\text { in } \mathrm{GeV}^{2}\right), & H_{0}=50 \\ 5.7 \times 10^{-7} / M_{\tilde{\nu}}^{2}\left(\text { in } \mathrm{GeV}^{2}\right), & H_{0}=100\end{cases}
$$

Hence sneutrino annihilation leads to a substantial peak in the background neutrino flux at an energy $E_{v}=M_{i}$. The obvious place to look for this type of monochromatic neutrino flux is in proton decay experiments. For example, the IMB detector observes roughly one event per day for a neutrino flux of about 0.25 $\left(\mathrm{cm}^{2} \mathrm{~s} \mathrm{sr}\right)^{-1}$ in this energy range [21]. If we optimistically estimate that the observation of one event per 100 days would be sufficient to reveal a monochromatic neutrino flux, this would correspond to an experimental sensitivity of about $2.5 \times$ $10^{-3}\left(\mathrm{~cm}^{2} \mathrm{~s} \mathrm{sr}\right)^{-1}$. Unfortunately, this is still several orders of magnitude larger than our theoretical expectations (17), so the prospect of observing monochromatic neutrinos from annihilating sneutrino dark matter appears remote.

In this paper we have explored the phenomenology of supersymmetric dark matter, following ref. [1], for both the general neutralino and the sneutrino LSP scenarios. We have found that in the neutralino case the requirement of closure admits two distinct solutions - either the LSP is a relatively pure photino or a relatively pure higgsino. Both of these neutralino scenarios can lead to observable antiproton, positron and gamma-ray fluxes. They are most easily distinguished by the ratio of $\mathrm{e}^{+} / \overline{\mathrm{p}}$ fluxes, which is larger in the photino case and relatively free of the uncertainties associated with the accretion of neutralinos and the trapping of cosmic rays in the galactic halo. We have also noted that photino scenarios leading to observable fluxes (i.e., those with light sfermion masses) can be tested in $\mathrm{e}^{+} \mathrm{e}$ experiments via the radiative production of photinos. A negative result from such experiments could be interpreted as evidence in favor of the higgsino LSP scenario - provided cosmic ray $\overline{\mathrm{p}}$ data persists at a level near $F_{\overline{\mathrm{p}}} \sim 3 \times 10^{-6}$ $\left(\mathrm{cm}^{2} \mathrm{~s} \mathrm{sr}\right)^{-1}$. Indeed, if the large $\overline{\mathrm{p}}$ flux of [13] is confirmed and is not due to conventional mechanisms, only scenarios \#1, \#9, and conceivably \#2, \#5, and \# 6 could be correct. Thus, for example, if the analyses of ref. [9] based on the CERN monojet data [22] ultimately require squark masses of order $100 \mathrm{GeV}$, only scenario \#9 would remain.

We have also shown that the sneutrino LSP scenario is restricted by the closure requirement to sneutrino masses less than $4 \mathrm{GeV}$. Sneutrino dark matter leads to no appreciable $\overline{\mathrm{p}}, \mathrm{e}^{+}$or $\gamma$-ray fluxes but results in a monochromatic neutrino flux that is probably unobservable. 
We appreciate conversations with G. Goldhaber, J. Hassard, G. Parle, J. Silk, and J. Stone.

\section{References}

[1] J. Silk and M. Srednicki, Phys. Rev. Lett. 53 (1984) 624

[2] A. Guth, Phys. Rev. D23 (1981) 347

[3] J. Yang, M. Turner, G. Steigman, D. Schramm and K. Olive, Ap. I., to be published; D. Hegyi and K. Olive, Phys. Lett. 126B (1983) 28

[4] C.J. Hogan, N. Kaiser, M.S. Turner and N. Vittorio, FERMILAB-Conf-85/57-A

[5] J. Ellis, J.S. Hagelin, D.V. Nanopoulos, K. Olive and M. Srednicki, Nucl. Phys. B238 (1984) 453

[6] J.S. Hagelin, G.L. Kane and S. Raby, Nucl. Phys. B241 (1984) 638;

L.E. Ibanez, Phys. Lett. 137B (1984) 160

[7] K. Inoue, A. Kakuto, H. Komatsu and S. Takeshita, Prog. Theor. Phys. 68 (1982) 927:

L. Alvarez-Gaume, J. Polchinski and M.B. Wise, Nucl. Phys. B221 (1983) 495;

J. Ellis, J.S. Hagelin, D.V. Nanopoulos and K. Tamvakis, Phys. Lett. 125B (1983) 275 :

J.M. Frere and G.L. Kane, Nucl. Phys. B223 (1983) 331

[8] J. Ellis, J.S. Hagelin, D.V. Nanopoulos and K. Tamvakis, Phys. Lett. 125B (1983) 275

[9] R.M. Barnett, H.E. Haber and G.L. Kane, SLAC-PUB-3551 (1985);

J. Ellis and M. Sher, CERN-TH. 3968/84 (1984);

L.E. Ibanez, C. Lopez and C. Munoz, Nucl. Phys. B256 (1985) 218;

K. Enqvist, D.V. Nanopoulos and A.B. Lahanes, CERN-TH.4095/85 (1985);

L.J. Hall, R.L. Jaffe and J.L. Rosner, preprint CTP \#1202 (1984)

[10] M. Roos et al. (Particle Data Group), Phys. Lett. 111B (1982) 1;

G.S. Abrams et al., Phys. Rev. Lett. 44. (1980) 10:

J.L. Siegrist et al., Phys. Rev. D 26 (1982) 969;

M.W. Coles et al., Phys. Rev. D 26 (1982) 2190;

M.S. Alam et al., Phys. Rev. Lett. 51 (1983) 1143 and references therein;

G. Goldhaber, private communication (1985)

[11] J. Gunn, B. Lee, I. Lerche, D. Schramm and G. Steigman, Ap. J. 233 (1978) 1015

[12] V. Ginzburg and V. Ptuskin, Rev. Mod. Phys. 48 (1976) 161

[13] A. Buffington, S. Schindler and C. Pennypacker, Ap. J. 248 (1981) 1179

[14] R. Protheroe, Ap. J. 251 (1982) 387;

P. Kiraly, J. Szabelski, J. Wdowczyk and A.W. Wolfendale, Nature 293 (1981) 120;

F. Stecker, R. Prothero and D. Kazanas, Ap. Space Sci. 96 (1983) 171;

L.C. Tan and L.K. Ng, Ap. J. 269 (1983) 751

[15] R. Protheroe, Ap. J. 254 (1982) 391

[16] S. Stone, in Proc. of the Int. Symp. on Lepton and photon interactions at high energies (Cornell University, August, 1983) and references therein

[17] G. Parle, private communication (1985)

[18] P. Fayet, Phys. Lett. 117B (1982) 460;

J. Ellis and J.S. Hagelin, Phys. Lett. 122B (1983) 303;

K. Grassie and P.N. Pandita, Dortmund Univ. preprint DO-TH83/23 (1983);

T. Kobayashi and M. Kuroda, Phys. Lett. 139B (1984) 208;

J.D. Ware and M.E. Machacek, Northeastern Univ. preprint NUB-2626 (1984)

[19] D. Burke, R. Hollebeck, et al., PEP experiment in progress

[20] MAC Collaboration, presented at the Vanderbilt Conference, 1984

[21] IMB Collaboration, private communication (1985)

[22] G. Arnison, et al., Phys. Lett. 139B (1984) 115 